\title{
Advances in Optical Fiber Bragg Grating Sensor Technologies
}

\author{
A. Ping ZHANG, Shaorui GAO, Guofeng YAN, and Yinbing BAI \\ Center for Optical and Electromagnetic Research, State Key Laboratory of Modern Optical Instrumentation, Zhejiang \\ University, Hangzhou, 310058, China
}

*Corresponding author: A. Ping ZHANGＥ-mail: zhangap@zju.edu.cn

\begin{abstract}
The authors review their recent advances in the development of optical fiber Bragg grating (FBG) sensor technologies. After a brief review of the fiber grating sensors, several newly developed FBG sensors are described. With the continuous development of fiber materials, microstructures and post-processing technologies, FBG sensors are still creative after the first demonstration of permanent gratings thirty years ago.
\end{abstract}

Keywords: Fiber Bragg gratings, optical fiber flowmeter, refractive-index sensor, optical fiber gas sensor

\section{Introduction}

An optical fiber grating is a one-dimensional periodic modulation of the refractive index which is normally formed based on the fiber photosensitivity. Because of its special phase-matching resonant mechanism, optical fiber grating has been proved as ideal in-fiber element for wavelength-selective lightwave reflection and filtering in optical fiber communications and sensing applications [1-4].

Fiber gratings are intrinsically sensing elements as their resonant wavelengths vary with the perturbation induced change of the grating period and the fiber effective index. Although the conventional electro-mechanical sensors were well established and are still under continuously development, fiber grating sensors have exhibited their distinct advantages and competitiveness in developing e.g. sensor array multiplexed in serial fashion, remote sensors for harsh environment monitoring, and ultrasensitive miniature optical sensors, due to their well known merits including electrically passive operation, immunity of electromagnetic interference, self-referencing and multiplexing capabilities, miniature size and high sensitivity.

Both fiber Bragg gratings (FBGs) and long-period gratings (LPGs), classified by their different mode coupling behaviors, have been demonstrated for the design and realization of a wide variety of sensors, e.g. strain, temperature, pressure, bending, vibration, and refractive index sensors [4-8]. If incorporated with functional coating and evanescent wave sensing technologies, the fiber grating sensors can be further developed as humidity, gas, chemical, and biochemical sensors [9-11].

With the continuous development of fiber materials and (micro)structures, optical fibers have become not only an optical waveguide but also an ideal platform for interdiscipline and multifunction integration. Due to their unique spectral and dispersion characteristics, grating structures will play a significant role in the development of advanced fiber-based devices, e.g. lab in a fiber.

In this review, we present our recent advances in the development of several new kinds of fiber

Received: 1 September 2011/ Revised version: 28 October 2011

(C) The Author(s) 2011.This article is published with open access at Springerlink.com 
grating sensors which include:

(1) All-fiber Bragg grating anemometer: a miniature optical fiber anemometer is proposed by using two laser-heated FBGs, and the speed of air flow is measured by determining the dynamic thermal equilibrium between the laser heating and air flow cooling through the monitoring of the FBG central wavelengths [12].

(2) Microstructured optical fiber (MOF) Bragg grating sensors: two new kinds of FBGs incorporated MOF sensors based on the evanescent-waves of guided modes are presented for microfluidic and gas sensing applications, respectively [13, 14].

(3) Microfiber Bragg grating sensor: a microfiber Bragg grating with a diameter of several micrometers is demonstrated for ultrasensitive sensing applications.

\section{All-fiber Bragg grating anemometer}

Electrical hot-wire anemometry was invented several decades ago and it still serves as an indispensable tool in the flow measurement field. Although many new kinds of principles [15-18] were proposed for air-speed or flow measurement, e.g. laser Doppler technology $[15,16]$, cross-correlation technique [17] or fiber modal interferometer [18], the classical hot-wire anemometry is still an irreplaceable approach for flow speed measurement particularly for the investigation of rapidly varying flow and turbulence [19].

The working principle of hot-wire anemometry (HWA) lies on the air-flow induced heat transfer from sensors to the surrounding environment. For instance, D. W. Lamb et al. proposed a low-cost FBG anemometer externally heated by using a $\mathrm{CO}_{2}$ laser for gas flow measurement in the high-voltage environment (corona discharge) [20]. C. Jewart et al. presented a flowmeter based on two cross-mounted FBGs with the thin-silver coating as the heat source after pumping by laser light. This flowmeter can measure the magnitude and direction of gas flow simultaneously [21]. Compared with electrical HWA, the laser heated FBG-based anemometer has some enhanced features like electromagnetic immunity and multiplexing capability.

We demonstrated a new all-optical fiber anemometer based on a two-FBG concatenated structure in which a laser beam is launched into the fiber and heats the FBG internally [12]. Figure 1 shows the schematic diagram of the proposed FBG anemometer in which two FBGs (S-FBG and $\mathrm{R}-\mathrm{FBG}$ ) written in the optically heatable fiber (OHF) are utilized as sensing and reference elements, respectively. The pump laser is launched into the fiber core thus the temperature of the FBGs will raise through the nonradiative absorption [22]. Broadband light whose wavelength differs from that of the pump laser is used as the probe light to monitor the Bragg wavelength of the laser-heated FBGs (LHFBGs). By monitoring the Bragg wavelength of the S-FBG, one can deduce the air flow induced heat loss. The R-FBG is packaged inside a section of the heat-insulation tube for evaluating the laser power variation.

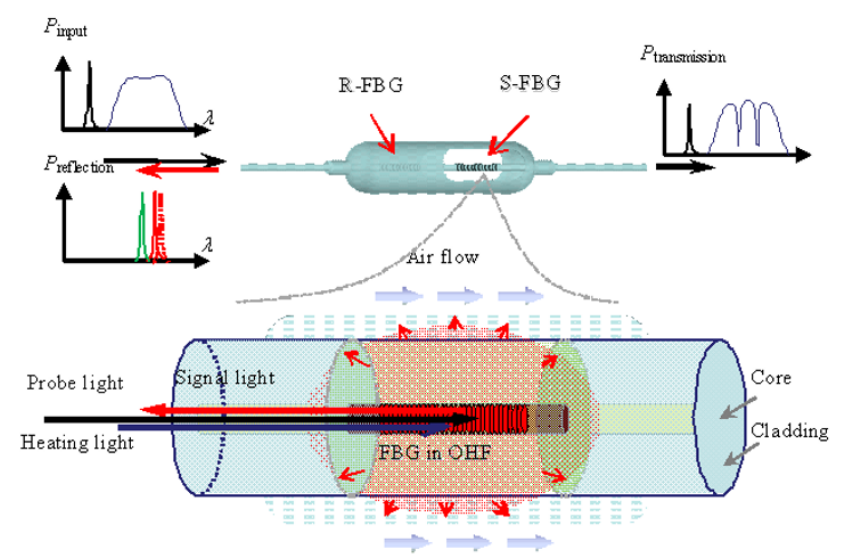

Fig. 1 Schematic diagram and working principle of the all-optical fiber anemometer based on laser-heated fiber Bragg gratings.

S-FBG and R-FBG used in the wind speed measurement experiment were inscribed in two light absorption fibers fabricated by CorActive High-Tech Inc., with different absorption coefficients. The S-FBG was inscribed in the section of the 
7-mm-length fiber with a higher light absorption coefficient $(5 \mathrm{~dB} / \mathrm{cm}$ at the $1480-\mathrm{nm}$ pump wavelength) thus could be heated to a higher temperature to achieve a larger dynamic range during the measurement. Another section of the 7-mm-length fiber with a lower absorption coefficient $(1 \mathrm{~dB} / \mathrm{cm}$ at the $1480-\mathrm{nm}$ pump wavelength) was used for fabricating the R-FBG. All two FBGs were fabricated with a 193-nm ArF excimer laser (Coherent, Bragg Star S-Industrial) and a phase mask whose period was $1070 \mathrm{~nm}$.

Figure 2 shows the evolution of spectra of the two LHFBGs pumped by using a 1480-nm semiconductor pump laser. As the pump power increases, both reflection peaks of the two LHFBGs shift toward the longer wavelength. The S-FBG exhibits a larger wavelength shift because of its higher absorption coefficient and therefore is heated more efficiently than the R-FBG. When the power of the pump laser increases from 0 to $280 \mathrm{~mW}$, the central wavelength of the S-FBG linearly shifts from $1546.24 \mathrm{~nm}$ to $1548.32 \mathrm{~nm}$, while the central wavelength of the R-FBG only shifts from $1544.56 \mathrm{~nm}$ to $1545.17 \mathrm{~nm}$. Assuming the total loss of the two fusion splices and the 7-mm-length low light-absorption fiber is about $1 \mathrm{~dB}$, the optical power of the pump light reaching the S-FBG is about $80 \%$ of that reaching the R-FBG. One can thus deduce that the pump power consumed by the S-FBG is 3 times more than that consumed by the R-FBG.

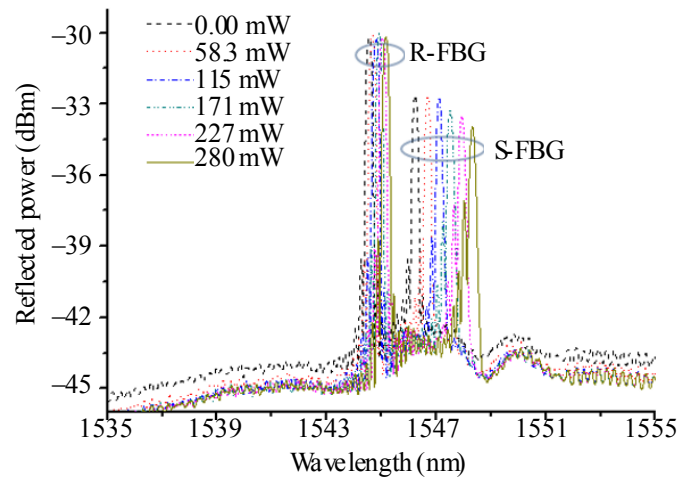

Fig. 2 Evolution of reflection spectra of the LHFBGs under heating of the $1480-\mathrm{nm}$ pump laser.
In order to minimize the heat transfer, the R-FBG was sealed inside a thin alundum tube with an inner diameter of $0.6 \mathrm{~mm}$ and outer diameter of $2 \mathrm{~mm}$, as illustrated in Fig. 1. The packaged R-FBG and the unpackaged S-FBG were installed inside a specially designed stainless steel tube whose inner diameter and outer diameter were $3 \mathrm{~mm}$ and $4 \mathrm{~mm}$, respectively. Convection slots were made in the stainless steel tube in both sides to allow the S-FBG to be exposed to the open air. All two LHFBGs and fiber were bonded inside the tubes by epoxy adhesive carefully.

A commercial wind tunnel (SANLING KQD-03, Scientific \& Teaching Instruments Factory of ECNU) and an electrical HWA (DANTEC, Stream Line 90N10 Frame) were utilized to evaluate the performance of the FBG-based anemometer. The wind speed could be tuned from 0 to $8.0 \mathrm{~m} / \mathrm{s}$. The FBG-based anemometer was fixed around the outlet while the electrical anemometer was also fixed for calibration. It was estimated that the same wind speed passed around two anemometers. The 1480-nm laser light was launched into the FGB-based anemometer through a 1480/1550 wavelength-division multiplexer (WDM). Both LHFBGs were interrogated by an FBG interrogator whose resolution and sampling frequency were $1.0 \mathrm{pm}$ and $25 \mathrm{~Hz}$, respectively.

In the experiments, the wind speed in the wind tunnel was manually adjusted, and the minimum wind speed that could be set by the wind tunnel was around $1.5 \mathrm{~m} / \mathrm{s}$. Figure 3 shows the measured sensor responses to different wind speeds and different pump powers. When the power of pump laser is $84.05 \mathrm{~mW}$ and no wind flows, the central wavelengths of the R-FBG and the S-FBG are $1543.803 \mathrm{~nm}$ and $1546.747 \mathrm{~nm}$, respectively. As wind speed increases from 0 to $8 \mathrm{~m} / \mathrm{s}$, the central wavelength of the S-FBG shifts to the shorter wavelength, while the central wavelength of the R-FBG is almost steady. The sensitivity of the FBG-based anemometer is relatively high when the 
wind speed is low and becomes lower at high wind speed. The FBGs exhibit similar responses when the power of the pump laser is changed to $195.1 \mathrm{~mW}$ and $299.3 \mathrm{~mW}$, respectively.

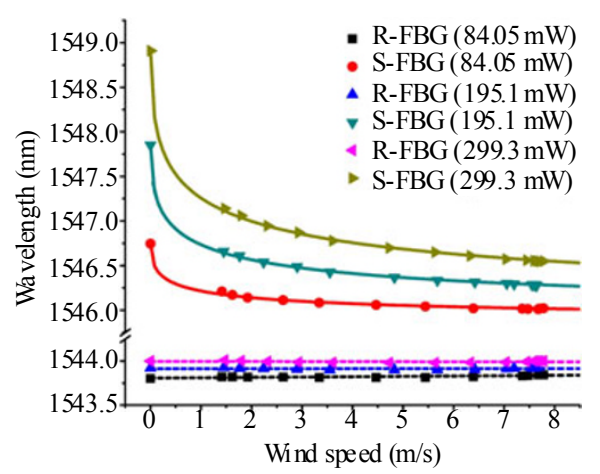

Fig. 3 Measured central wavelengths versus the wind speed of the FBG-based anemometer under different pumping powers: the solid curves are fitted by using the derived equation.

Based on the theory of the hot-wire anemometer [19], the relationship between the heat loss, $H_{\text {loss }}$, and the wind speed, $v$, can be written as

$$
H_{\text {loss }}=\left[T_{\mathrm{a}}(v)-T_{\mathrm{e}}\right](A+B \sqrt{v})
$$

where $T_{\mathrm{a}}$ is the temperature of the anemometer, $T_{\mathrm{e}}$ is the temperature of the environment, $A$ and $B$ are empirical calibration constants. Based on the rule of energy conversation, the heat loss should be equal to the power consumption of the S-FBG, i.e.

$$
H_{\text {loss }}=P_{\text {input }}\left(1-a_{\mathrm{r}}\right) a_{\mathrm{s}}
$$

where $P_{\text {input }}$ is the input laser power, $a_{\mathrm{r}}$ is the absorption coefficient of the R-FBG, and $a_{\mathrm{s}}$ is the absorption coefficient of the S-FBG.

Moreover, the dependence of the wavelength shift of the FBG, $\Delta \lambda$, on the change of the temperature, $\Delta T=T_{a}(v)-T_{a}(0)$, is well known as

$$
\Delta \lambda / \lambda_{\mathrm{h} 0}=(\alpha+\xi) \Delta T
$$

where $\lambda_{\mathrm{h} 0}$ is the wavelength of the heated FBG before wind blowing, $\alpha$ is the thermal expansion coefficient, and $\xi$ is the thermo-optic coefficient. Using (1) and (3), the dependence of the wavelength shift of the FBG anemometer on the wind speed can be deduced as

$$
\Delta \lambda=\lambda_{\mathrm{h} 0}(\alpha+\xi)\left[H_{\text {loss }} /(A+B \sqrt{v})-\Delta T_{0}\right]
$$

where $\Delta T_{0}=T_{\mathrm{a}}(0)-T_{\mathrm{e}}$. If $\lambda_{\mathrm{e} 0}$ is defined as the wavelength of the FBG before heating, the above equation can be further written as

$$
\lambda(v)-\lambda_{\mathrm{e} 0}=\lambda_{\mathrm{h} 0}(\alpha+\xi) H_{\text {loss }} /(A+B \sqrt{v}) .
$$

The solid curves in Fig. 3 show the fitted results using the above equation. One can see that the experimental results agreed very well with the theory of the hot-wire anemometer.

The sensitivity of the sensor depends on the heat transfer rate from the sensor to the surrounding environment because HWA is based on the heat-transfer principle. An anemometer was recoated with epoxy to evaluate the coating's effects on the sensor performance. The diameter of the recoated fiber was about $0.5 \mathrm{~mm}$. In order to evaluate the effects of pump power, the LHFBGs were pumped at three different powers with the 1480-nm laser.

Due to the non-linear responses of the anemometer in the range of wind speed between $2.0 \mathrm{~m} / \mathrm{s}$ and $8.0 \mathrm{~m} / \mathrm{s}$, as shown in Fig. 3, slopes of linearly fitted data are calculated as the effective sensitivity to evaluate the effects of the pump power and the recoating on the sensor performance. Figure 4 presents the calculated effective sensitivities of the two anemometers with and without coating. One can see that the sensitivities of the two anemometers increase with an increae of the pump power. As expected, the recoating process can be used to reduce the sensitivity of the anemometer. Based on the calculated sensitivity and the resolution of the FBG interrogator, i.e. $1 \mathrm{pm}$, it can be deduced that the resolution of wind speed measurement with the anemometer is about $0.012 \mathrm{~m} / \mathrm{s}$ for the wind speed ranging from $2 \mathrm{~m} / \mathrm{s}$ to $8 \mathrm{~m} / \mathrm{s}$.

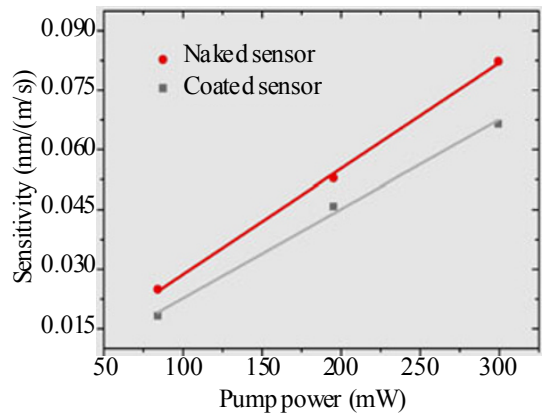

Fig. 4 Effective sensitivities of the recoated and naked anemometers at different pump powers. 


\section{Microstructured optical fiber Bragg grating sensors}

MOFs with numerous air-holes along the length of the cladding have attracted wide interest in recent years. The unique holey cladding structure offers marvellous benefits as liquid or gas cells, because of which MOFs provide an ideal platform for light-matter interaction and sensing applications [23-26].

In the last few years, researchers have paid particular attentions to grating incorporated MOF sensors, because of their unique spectral and coupling properties. For instance, FBG incorporated MOF sensors can induce a contra-directional coupling between forward and backward fiber modes through resonant scattering and thus perform very well in strain, pressure and microfluidic refractive index sensing applications [27-32]. For instance, C. Martelli et al. characterized strain and temperature responses of the FBG on the MOF and showed an increased sensitivity to strain by leaky modes of MOFs which was not presented in conventional fibers [27]. C. Jewart et al. demonstrated that the transverse stress sensitivity of the two-hole fiber could be enhanced by a factor of eight compared with a standard fiber [28]. T. Martynkien et al. designed, manufactured, and characterized two kinds of birefringent MOFs. The sensitivity to the hydrostatic pressure of both fibers exceeded $-43 \mathrm{rad} / \mathrm{MPa} \bullet \mathrm{m}$ at $1.55 \mu \mathrm{m}$ [29]. M. C. P. Huy et al. inscribed the common FBG on three-hole MOFs [30] and inscribed titled FBG on six-hole MOFs [31]. The refractive index resolution of both sensors could reach $7 \times 10^{-6}$ and $6.8 \times 10^{-6}$, respectively.

Moreover, numerous tunable optical fiber devices were demonstrated based on FBG incorporated MOFs. For instance, C. Kerbage et al. presented two methods for manipulating light in MOFs by displacing and positioning micro-fluids along the air-holes of the fiber $[33,34]$.

\subsection{Microstructured optical fiber Bragg grating microfluidic sensor}

Although numerous conventional fiber devices, e.g. LPGs or tilted FBGs (TFBGs), were proposed for refractive index measurement, MOFs provide a new platform for light-matter interaction and refractive index (RI) measurement of much less volume of the measurand. MOFs with gratings including long period gratings (LPGs) and FBGs have been demonstrated for developing highly sensitive microfluidic RI sensors [35-38]. However, all proposed MMOF Bragg grating (MOFBG) sensors are using the large-hole MOF and thus not ideal for e.g. surface enhanced Raman scattering sensors.

We proposed a specially designed MOFBG sensor with a large number of small holes [13], as shown in Fig. 5. The core of the MOF is highly doped with germanium for grating fabrication. Such an MOF dramatically increases inner surface and thus can be used for developing ultrasensitive sensors through further the deposition of the sensing nanocoating in the micro-channels.

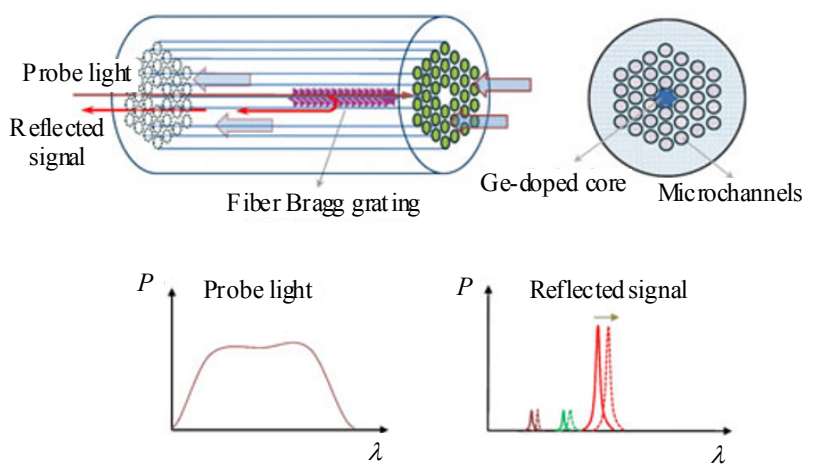

Fig. 5 Schematic diagram of the microstructured optical fiber Bragg grating as a microfluidic sensor.

The scanning electron microscope (SEM) images of the MOF are shown in Fig. 6, from which we can see that the core with a diameter of about $10.5 \mu \mathrm{m}$ is surrounded with 3.45 - $\mu \mathrm{m}$-diameter holes, and the center to center intervals of the holes are about $3.73 \mu \mathrm{m}$. Since the ratio of the hole diameter to pitch is relatively large, this kind of MOF is multimode around $1550 \mathrm{~nm}$. 


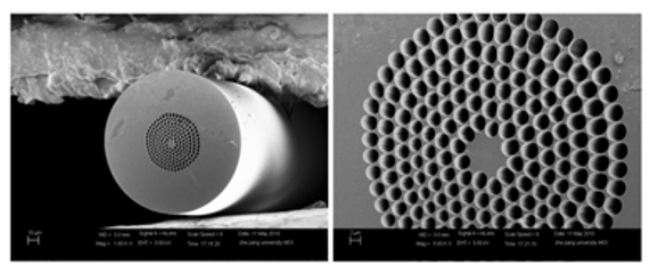

Fig. 6 SEM images of the microstructured optical fiber used in the experiments.

Before the fabrication of the FBG, the MOF is fusion spliced with standard optical fibers and hydrogen loaded at $10 \mathrm{MPa}$ and $100{ }^{\circ} \mathrm{C}$ for three days to enhance the photosensitivity. Then Bragg gratings are written using a 248 Excimer laser (Bragg Star 200, TuiLaser) with a phase mask whose period is $1070.6 \mathrm{~nm}$. A reflective spectrum of a $10-\mathrm{mm}$ FBG measured is shown in Fig. 7. There are four significant reflection peaks at $1527.83 \mathrm{~nm}$, $1530.69 \mathrm{~nm}, \quad 1531.0 \mathrm{~nm}$ and $1547.55 \mathrm{~nm}$, respectively. By using a numerically simulation with the commercial beam propagation method software (RSoft Design Group), it is deduced that the reflection peak at $1547.55 \mathrm{~nm}$ corresponds to the fundamental mode, the other two reflection peaks at $1531.0 \mathrm{~nm}$ and $1530.69 \mathrm{~nm}$ correspond to the two polarizations of the second mode, and the forth peak at $1527.83 \mathrm{~nm}$ corresponds to the third mode of the MOF. The patterns of the first three orders of modes are shown in the insets of Fig. 7.

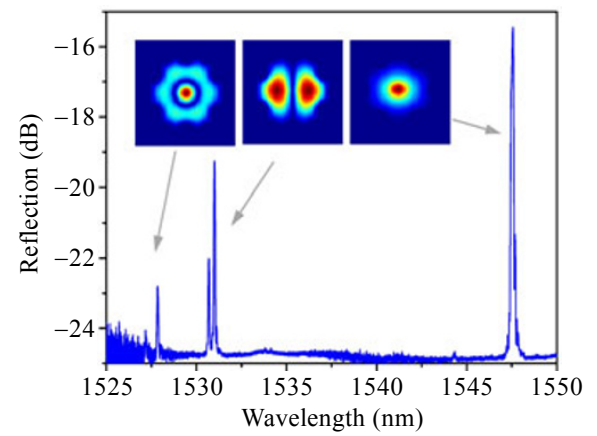

Fig. 7 Reflection spectrum of the fabricated microstructured optical fiber Bragg grating (the insets show the simulated mode patterns of corresponding reflection peaks).

The responses of such an MOFBG to the refractive index in the micro-channels were experimentally and numerically investigated. We prepared the sucrose solutions with different concentrations [0,15.023, 29.577, 40.002, 45.031, 49.998 and 59.893 (mass\%)], which RIs are 1.3330, $1.3557,1.3812,1.3999,1.40978,1.4201$ and 1.4407 , respectively [39], for the sensor tests. A vacuum pump was used in our experiments for quickening the filling process. Before changing samples, the MOF was purged by using the vacuum pump to depress the measurement errors. A probing fiber was mounted on a 3-axis translation stage to couple the light into the MOF and collect the light reflected back from the MOF as well.

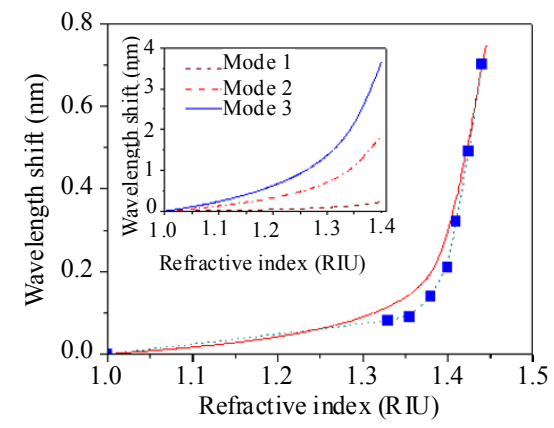

Fig. 8 Reflection spectra of the fabricated microstructured optical fiber Bragg grating (the inset shows the simulated mode patterns of corresponding reflection peaks).

Figure 8 shows the comparison of the measured and calculated results of the responses of the fundamental mode to the change of RIs at the room temperature $\left(\sim 22{ }^{\circ} \mathrm{C}\right)$. One can see that the experimental results agree well with that of the simulation. In the RI range of 1.31 to 1.38 , the Bragg wavelength of the fundamental mode is not so sensitive to the change in RIs; whereas in the RI range of 1.4 to 1.44 , the Bragg wavelength exhibits the significant sensitivity. The responses of the higher order modes are numerically calculated and presented in the inset of Fig. 8. The sensitivities of the first three order modes are $15 \mathrm{~nm} / \mathrm{RIU}$, $21 \mathrm{~nm} / \mathrm{RIU}$, and $45 \mathrm{~nm} / \mathrm{RIU}$, respectively, in the RI range of 1.4 to 1.44 . Assuming that the resolution of the measurement of the Bragg wavelength can reach 1 picometer, it can be deduced the minimum detectable RI change of the MOFBG sensor is from $2.2 \times 10^{-5}$ to $6.7 \times 10^{-5}$, depending on the order of fiber 
modes. It is expected that the higher sensitivity can be achieved if the MOF parameters are further optimized through reducing the core area to make the MOF single-mode operation and enhance the evanescent wave.

\subsection{Microstructured optical fiber gas sensor}

The MOF is inherent a gas cell which could have much longer light-gas interaction length than conventional gas cells. In the past few years researchers have proposed many different designs to carry out gas spectroscopy in MOFs which can be mainly classified into two types of structures. One is to employ hollow core MOFs to maximize or enhance the overlap between guided light and gas. Y. L. Hoo et al. firstly proposed gas diffusion measurement using the hollow-core photonic bandgap fiber [40], almost at the same time T. Ritari et al. addressed a paper on gas sensing using the air-guided photonic bandgap fiber [26]. The other one is to use solid core or suspended core MOFs [41-43] in which the light is guided by total internal reflection and interacts with samples via evanescent waves. For example, G. Pickrell et al. proposed a process to sense gas with random-hole optical fiber through evanescent-wave absorption [42].

However, all those demonstrated MOF gas sensors work in transmission mode and thus are not convenient for some practical applications. Introducing FBGs into MOFs can not only change the operation mode, but also improve the detection selectivity of the gas sensor. To the best of our knowledge, for the first time we demonstrated a design and fabrication of an MOFBG for gas sensing applications [14].

The designed MOFBG gas sensor is shown in Fig. 9. The Bragg wavelength of the MOFBG has been appropriately set to overlap a characteristic absorption peak of the acetylene $\left(\mathrm{C}_{2} \mathrm{H}_{2}\right)$ gas in the near-IR range [44]. In order to suppress the effects of light source fluctuation and propagation loss on measurement results, an FBG written in the standard single-mode fiber (SMF) is spliced with the
MOFBG as a reference element (RFBG). Therefore, the light intensity at the Bragg resonance wavelength will be modulated by the gas in holes through evanescent-wave absorption.

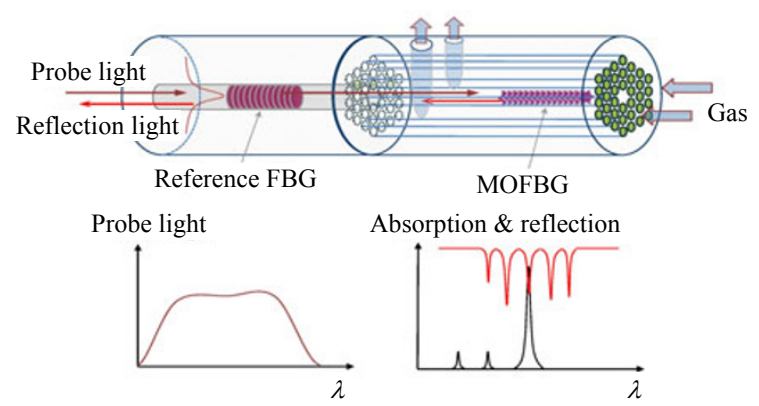

Fig. 9 Reflection spectra of the fabricated microstructured optical fiber Bragg grating (the insets show the simulated mode patterns of corresponding reflection peaks).

If the gas is assumed to fill out the MOF, the intensity of the reflected light, $I_{\mathrm{R}}$, can be written as

$$
I_{\mathrm{R}}=R I_{0} \exp [-2 r \alpha(\lambda) C L]
$$

where $I_{0}$ is the intensity of input light at the wavelength of the absorption line of the gas, $R$ is the reflectivity of the MOFBG (including the coupling loss between the SMF and MOF), $\alpha$ is the absorption coefficient of the gas at wavelength $\lambda$, $C$ is the concentration of the gas, $L$ is the length of the gas cell (including the half length of the MOFBG), and $r$ is the relative sensitivity coefficient of the evanescent wave [45].

We use the normalized absorption of light in decibel as $A=-10 \log _{10}\left(I_{R} / R I_{0}\right)$ to express the absorption as

$$
A(\mathrm{~dB})=8.7 r \alpha(\lambda) C L .
$$

It reveals that the light absorption in decibel linearly depends on the concentration of the gas, and its sensitivity mainly depends on the absorption coefficient of the gas at the corresponding wavelength and the length of the gas cell for a given fiber mode.

The Bragg gratings are still written by using phase mask technique but with a 193-nm Excimer laser (Bragg Star Industrial 1000, Coherent). The period of the phase mask used here in the grating fabrication is $1070.0 \mathrm{~nm}$. The gratings are fabricated 
without a hydrogen loading process. Figure 10 shows the measured reflection spectra of the sensing FBG written in the MOF and the reference FBG written in the standard SMF. One can see that there are five reflection peaks in the spectra. The highest reflection peak at $1548.23 \mathrm{~nm}$ corresponds to the Bragg wavelength of the FBG written in a standard SMF. The other four reflection peaks are formed with the fabrication of the MOFBG. The peak at $1545.76 \mathrm{~nm}$ corresponds to the reflected fundamental mode of the MOF, the two peaks at $1529.21 \mathrm{~nm}$ and $1528.94 \mathrm{~nm}$ correspond to the two polarizations of the second order mode, and the reflection peak at $1525.37 \mathrm{~nm}$ corresponds to the third order mode of the MOFBG. Since the evanescent wave of the higher order mode is stronger than that of lower order modes, the grating period of the MOFBG has been appropriately selected so that the Bragg wavelength of the second order fiber mode is located at the significant absorption line of the $\mathrm{C}_{2} \mathrm{H}_{2}$ gas, i.e., $1529.21 \mathrm{~nm}$. The modal pattern of this sensing mode is presented in the inset of Fig. 10, from which the fraction of energy of the field in the air holes is calculated as $12 \%$.

The MOFBG sensor was tested and compared with the above analysis. The length of the sensor was about $3.5 \mathrm{~cm}$, and its sensing peak overlapped with the $\mathrm{C}_{2} \mathrm{H}_{2}$ absorption line at $1529.21 \mathrm{~nm}$ (the reflection spectra are shown in Fig. 10). According to the $\mathrm{C}_{2} \mathrm{H}_{2}$ absorption spectra (Hitran Database), the absorption coefficient of the $\mathrm{C}_{2} \mathrm{H}_{2}$ gas at this wavelength is $49.87 / \mathrm{m}$.

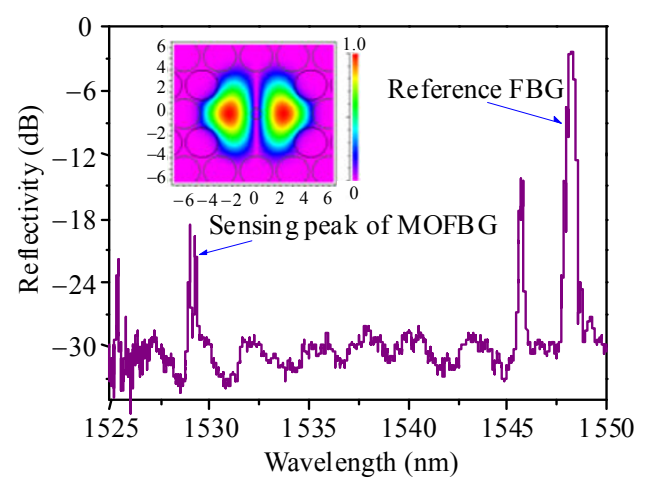

Fig. 10 Reflection spectra of the fabricated MOFBG sensor (the inset shows the simulated modal pattern of the sensing mode).
A home-made chamber connected with a vacuum pump and gas cylinders was used for the sensor test. In the beginning, the chamber was pumped down to about $1 / 400 \mathrm{~atm}$ at a rate of $24 \mathrm{~L} / \mathrm{s}$. Then a specific amount of acetylene gas was filled into the chamber whose pressure was controlled by a valve. Thereafter, nitrogen gas was added to keep the inside pressure at about $1 \mathrm{~atm}$. The above steps were repeated with adjustments of the amount of acetylene gas. The reflection spectra of the sensor were monitored with a broadband amplified spontaneous emission (ASE) light source and an optical spectral analyzer (OSA, ANDO, AQ6317).

The inset of Fig. 11 shows the response of the MOFBG sensor as the $\mathrm{C}_{2} \mathrm{H}_{2}$ gas concentration changes from $1.5 \%, 2 \%, 5 \%$, and $7 \%$, respectively. The reflected intensity of the reference FBG is utilized to normalize the absorption of the reflected signal. One can see that the absorption increases with an increase of the gas concentration and could fall down if the $\mathrm{C}_{2} \mathrm{H}_{2}$ gas is exhausted, as expected. The response time, $T_{\mathrm{R}}$, can be estimated about 6.5 minutes.

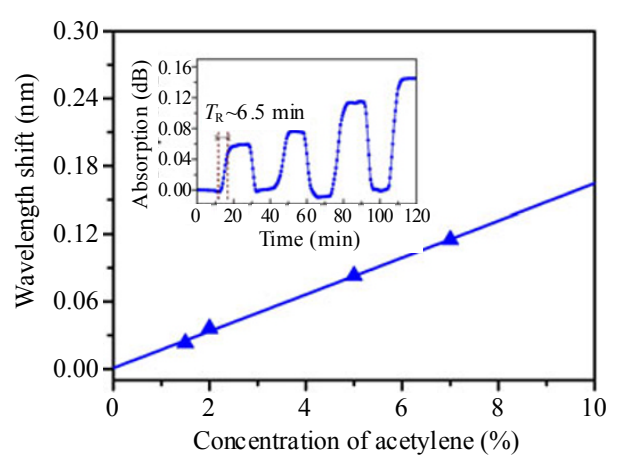

Fig. 11 Dependence of the absorptions of sensor (triangular points) on the $\mathrm{C}_{2} \mathrm{H}_{2}$ gas concentrations (the inset is the measurement responses of the sensor to the adjustment of $\mathrm{C}_{2} \mathrm{H}_{2}$ gas concentrations).

The dependence of the absorption of the sensor on the concentration of acetylene gas is presented in Fig. 11. Through a linear fitting process, the sensitivity of the sensor is determined as about $0.017 \mathrm{~dB} / \%$. From (2), it can be seen that the sensitivity of this MOFBG sensor depends on the 
product of the effective absorption coefficient at the reflection wavelength and the length of the gas cell. Based on the corresponding values of the sensor given above, the theoretical value of its sensitivity ratio is $0.015 \mathrm{~dB} / \%$. Such a difference might be induced by several factors:

(1) The reflection peak of the MOFGB might be slightly shifted and consequently can't exactly overlap the absorption line of $\mathrm{C}_{2} \mathrm{H}_{2}$ gas. Thus, the absorption coefficient might not be exactly as intended.

(2) The pressure control in the chamber is not ideal in the experiment which results in some reading errors with regard to the gas concentration.

Although the sensitivity of the sensor can be improved through the increment of the length of the gas cell, it will prolong the sensor's response time. So there lies a tradeoff between the response time and the sensor sensitivity. The side openings on the MOFs [46] or MOF with larger micro-holes can expedite sensor response.

It is noteworthy to propose such kind of MOFBG sensor which provides an ideal sensing technology based on absorption spectroscopy and is significantly potential in the fields of other gases, chemical or biological agent detection by using the similar method. Meanwhile, the higher order mode can also be used to improve the sensitivity because of its enhanced evanescent wave though it will increase a little bit of bending loss.

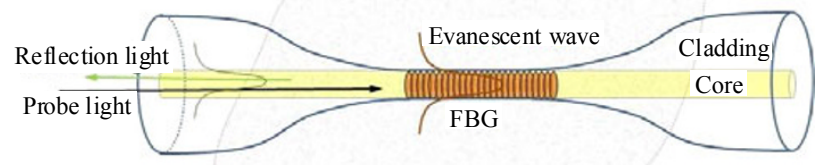

Fig. 12 Schematic diagram of the microfiber Bragg grating sensor for measuring surround media via evanescent wave.

The main potential problem of this MOFBG sensor is the possible measurement errors induced by temperature fluctuation. We proposed two solutions:

(1) Calibration approach. Since the wavelength of the reflection peak indicates the temperature, one can monitor both the wavelength shift and the intensity of the reflection peak to simultaneously obtain the gas induced absorption and temperature.

(2) Modulation scheme. One can use an electrically or optically heating method to modulate the wavelength of the MOFBG reflection peak around a particular absorption line and use the amplitude of the modulated reflection power, rather than the absolute value of the reflection power, to suppress the measurement errors induced by temperature fluctuations.

\section{Microfiber Bragg grating sensors}

Since the report of subwavelength-diameter silica wires for the low-loss optical wave guiding by L. M. Tong et al. [47], microfibers with a diameter of about several microns have become a topic of intense research interests due to the strong evanescent wave and high-intensity surface field [48]. Such a microfiber is particularly suitable for ultrasensitive sensing applications [49-53]. In recent years, researchers have paid a huge amount of attention to the fabrication of grating structures in the microfiber [54-58]. For instance, W. Jin et al. proposed the fabrication of long period gratings in optical microfibers by using $\mathrm{CO}_{2}$ laser pulses [54]. Microfiber Bragg gratings (MFBGs) were also demonstrated by wet chemical etch-erosion [55, 56], femtosecond laser writing [57] and focused ion beam milling technique [58]. For instance, Y. Liu et al. reported the fabrication of a highly compact MFBG with a length of $518 \mu \mathrm{m}$ and a diameter of $1.8 \mu \mathrm{m}$ by using focused ion milling technique, and a sensitivity of $660 \mathrm{~nm} / \mathrm{RIU}$ was achieved [58].

Such a microfiber Bragg grating is promising for developing miniature fiber-optic sensors with a high sensitivity and fast response. As shown in Fig. 12, the reflected light can be used to analyze the interaction between the evanescent wave and the surrounding medium. A small variation of the refractive index around the MFBG will induce a 
significant change in the Bragg wavelength according to the Bragg condition.

We demonstrated the fabrication of such an MFBG sensor by using a hydrofluoric acid (HF) etching procedure. The HF solution (20\%) was employed for etching after the FBG was written in the standard SMF-28 (Corning) fiber, which was mechanically stripped and cleaned with ethanol. The cladding etching rate of the used etchant was about $0.44 \mu \mathrm{m} / \mathrm{min}$. A protective liquid (isooctane) was used to prevent the evaporation of the HF solution.

Figure 13 shows the microscope images of the etched fibers obtained with different configurations. It can be seen that the transition region between the unetched part and the etched segment in Fig. 13 (a) is circularly symmetry, which illustrates the vibrations and turbulences during the experiment are very slight. The cone angle is mainly determined by the meniscus height of the liquid-liquid-solid interface circle directly after immersion under the static etching condition with a protection layer. Figure 13(b) shows the microfiber with a diameter of about $6.39 \mu \mathrm{m}$, with diminished surface corrugations. Another microfiber with a diameter of $3.38 \mu \mathrm{m}$ is shown in Fig. 13 (c), in which even periodic grating structure can be seen.

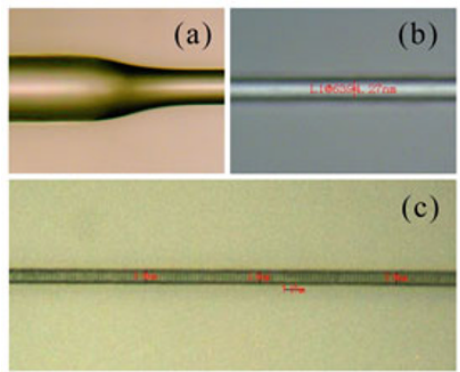

Fig. 13 Microscope images of the etched fiber with different configurations: (a) image of the transition region at the interface of the protecting overlayer and the HF solution, (b) and (c) images of the obtained microfiber.

The obtained microfiber Bragg grating was demonstrated in the RI sensing application. The diameter of the measured microfiber sensor was about $4 \mu \mathrm{m}$, and the self-prepared glucose solutions were used as test samples. Deionized water was applied to clean the residual glucose solution before a new measurement. Figure 14 shows the simulative and experimental results of such an MFBG response to the change in the refractive index of the surrounding medium. The Bragg wavelength increases with the increment of the refractive index, and good agreement between theory and experiment is achieved in the RI range of 1.33 to 1.40 . Using the slope of linearly fitted data as the effective sensitivity, it can be deduced that the RI sensitivity of the achieved microfiber grating sensor is about $82.4 \mathrm{~nm} /$ R.I.U. in the RI range of 1.418 to 1.4306 . Therefore, assuming that the detectable spectral resolution is $1 \mathrm{pm}$, it can be deduced that the minimum detectable refractive index resolution is $1.2 \times 10^{-5}$. In the RI range of 1.40 to 1.44 , the measured sensitivity is smaller than the simulative one due to the experimental errors. The calculated sensitivity of the MFBG in the RI of around 1.44 is about $343 \mathrm{~nm} / \mathrm{RIU}$. Such a high sensitivity is attributed to high-fractional evanescent fields around the MFBG.

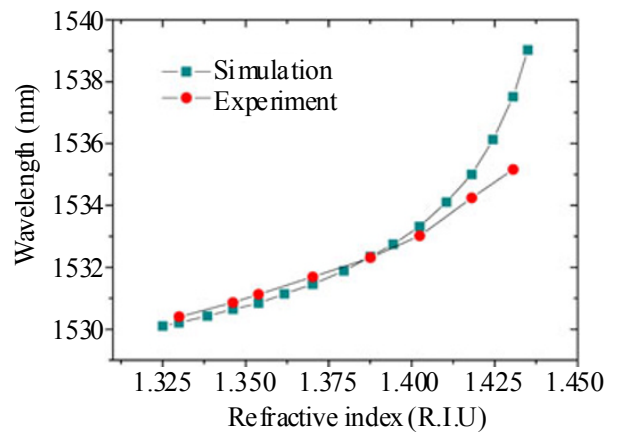

Fig. 14 Simulation and measurement of Bragg wavelength shifts with different surrounding refractive indices for the achieved MFBG sensor

\section{Conclusions}

In conclusion, we have reviewed some of our recent research advances on the design and fabrication of new FBG sensors. It has been demonstrated that optical fiber gratings are still playing a significant role for developing a wide 
variety of sensors with the fusion of new fiber materials and structures. Those new kinds of FBG sensors hold great potential for various industrial applications.

\section{Acknowledgement}

This work was supported by the Fundamental Research Funds for the Central Universities. The authors thanks Prof. H. Y. Tam of Hong Kong Polytechnic University, Prof. Y. Chung of Gwangju Institute of Science and Technology, Korea, and Prof. L. M. Tong of Zhejiang University for the research collaboration on the fiber grating anemometer, microstructured optical fibers, and microfiber sensors, respectively.

Open Access This article is distributed under the terms of the Creative Commons Attribution License which permits any use, distribution, and reproduction in any medium, provided the original author(s) and source are credited.

\section{References}

[1] K. O. Hill and G. Meltz, "Fiber Bragg grating technology fundamentals and overview," Journal of Lightwave Technology, vol. 15, no. 8, pp. 1263-1276, 1997.

[2] M. A. Davis, A. D. Kersey, H. J. Patrick, M. LeBlanc, K. P. Koo, C. G. Askins, M. A. Putnam, and E. J. Friebele, "Fiber grating sensors," Journal of Lightwave Technology, vol. 15, no. 8, pp. 1442-1463, 1997.

[3] C. R. Giles, "Lightwave applications of fiber Bragg gratings," Journal of Lightwave Technology, vol. 15, no. 8, pp. 1391-1404, 1997.

[4] Y. J. Rao, "In-fiber Bragg grating sensors," Measurement Science and Technology, vol. 8, no. 4, pp. 355-375, 1997.

[5] K. T. V. Grattan and T. Sun, "Fiber optic sensor technology: an overview," Sensors and Actuators A: Physical, vol. 82, no. 1-3, pp. 40-61, 2000.

[6] A. P. Zhang, B. O. Guan, X. M. Tao, and H. Y. Tam, "Experimental and theoretical analysis of fiber Bragg gratings under lateral compression," Optics Communications, vol. 206, no. 1-3, pp. 81-87, 2002.

[7] J. J. Zhu, A. P. Zhang, B. Zhou, F. Tu, J. T. Guo, W. J. Tong, S. He, and W. Xue, "Effects of doping concentrations on the regeneration of Bragg gratings in hydrogen loaded optical fibers," Optics Communications, vol. 284, no. 12, pp. 2808-2811, 2011.

[8] L. Y. Shao, A. P. Zhang, W. S. Liu, H. Y. Fu, and S. He, "Optical refractive-index sensor based on dual fiber-Bragg gratings interposed with a multimode-fiber taper," Photonics Technology Letters, vol. 19, no. 1, pp. 30-32, 2007.

[9] B. Gu, M. J. Yin, A. P. Zhang, J. W. Qian, and S. He, "Optical fiber relative humidity sensor based on FBG incorporated thin-core fiber modal interferometer," Optics Express, vol. 19, no. 5, pp. 4140-4146, 2011.

[10] X. T. Wei, T. Wei, H. Xiao, and Y. S. Lin, "Nano-structured Pd-long period fiber gratings integrated optical sensor for hydrogen detection," Sensors and Actuators B: Chemical, vol. 134, no.2, pp. 687-693, 2008.

[11] J. M. Corres, I. del Villar, I. R. Matias, and F. J. Arregui, "Fiber-optic pH-sensors in long-period fiber gratings using electrostatic self-assembly," Optics Letters, vol. 32, no. 1, pp. 29-31, 2007.

[12] S. R. Gao, A. P. Zhang, H. Y. Tam, L. H. Cho, and C. Lu, "All-optical fiber anemometer based on laser heated fiber Bragg gratings," Optics Express, vol. 19, no. 11, pp. 10124-10130, 2011.

[13] A. P. Zhang, G. F. Yan, S. R. Gao, S. He, B. Kim, J. Im, and Y. Chung, "Microfluidic refractive-index sensors based on small-hole microstructured optical fiber Bragg gratings," Applied Physics Letters, vol. 98, no. 22, pp. 221109, 2011.

[14] G. Yan, A. P. Zhang, G. Ma, B. Wang, B. Kim, J. Im, S. He, and Y. Chung, "Fiber-optic acetylene gas sensor based on microstructured optical fiber Bragg gratings," Photonics Technology Letters, vol. 23, no. 21, pp. 1588-1590, 2011.

[15] M. Stieglmeier and C. Tropea, "Mobile fiber-optic laser Doppler anemometer," Applied Optics, vol. 31, no. 21, pp. 4096-4105, 1992.

[16] G. D. Byrne, S. W. James, and R. P. Tatam, "A Bragg grating based fiber optic reference beam laser Doppler anemometer," Measurement Science and Technology, vol. 12, no. 7, pp.909-913, 2001.

[17] S. Takashima, H. Asanuma, and H. Niitsuma, "A water flowmeter using dual fiber Bragg grating sensors and cross-correlation technique," Sensors and Actuators A: Physical, vol. 116, no. 1, pp. 66-74, 2004.

[18] O. Frazao, P. Caldas, F. M. Araujo, L. A. Ferreira, and J. L. Santos, "Optical flowmeter using a modal interferometer based on a single nonadiabatic fiber taper," Optics Letters, vol. 32, no. 14, pp. 1974-1976, 2007.

[19] H. H. Bruun, Hot-wire anemometry: principles and signal analysis. Oxford: Oxford University Press, 1995. 
[20] D. W. Lamb and A. Hooper, "Laser-optical fiber Bragg grating anemometer for measuring gas flows: application to measuring the electric wind," Optics Letters, vol. 31, no. 8, pp. 1035-1037, 2005.

[21] C. Jewart, B. McMillen, S. K. Cho, and K. P. Chen, "X-probe flow sensor using self-powered active fiber Bragg gratings," Sensors and Actuators A: Physical, vol. 127, no. 1, pp. 63-68, 2006.

[22] M. Buric, K. P. Chen, M. Bhattarai, P. R. Swinehart, and M. Maklad, "Active fiber Bragg grating hydrogen sensors for all-temperature operation," IEEE Photonics Technology Letters, vol. 19, no. 5, pp. 255-257, 2007.

[23] A. Hassani and M. Skorobogatiy, "Design of the microstructured optical fiber-based surface plasmon resonance sensors with enhanced microfluidics," Optics Express, vol. 14, no. 24, pp. 11616-11621, 2006.

[24] D. K. Wu, B. T. Kuhlmey, and B. J. Eggleton, "Ultra-sensitive photonic crystal fiber refractive index sensor," Optics Letters, vol. 34, no. 3, pp. 322-324, 2009.

[25] F. Benabid, F. Couny, J. C. Knight, T. A. Birks, and P. S. Russell, "Compact, stable and efficient all-fiber gas cells using hollow-core photonic crystal fibers," Nature, vol. 434, no.7032, pp. 488-491, 2005.

[26] T. Ritari, J. Tuominen, H. Ludvigsen, J. Petersen, T. Sørensen, T. Hansen, and H. Simonsen, "Gas sensing using air-guiding photonic bandgap fibers," Optics Express, vol. 12, no. 17, pp. 4080-4087, 2004.

[27] C. Martelli, J. Canning, N. Groothoff, and K. Lyytikainen, "Strain and temperature characterization of photonic crystal fiber Bragg gratings," Optics Letters, vol. 30, no. 14, pp. 1785-1787, 2005.

[28] C. Jewart, K. P. Chen, B. McMillen, M. M. Bails, S. P. Levitan, J. Canning, and I. V. Avdeev, "Sensitivity enhancement of fiber Bragg gratings to transverse stress by using microstructural fibers," Optics Letters, vol. 31, no. 15, pp. 2260-2262, 2006.

[29] T. Martynkien, G. Statkiewicz-Barabach, J. Olszewski, J. Wojcik, P. Mergo, T. Geernaert, C. Sonnenfeld, A. Anuszkiewicz, M. K. Szczurowski, K. Tarnowski, M. Makara, K. Skorupski, J. Klimek, K. Poturaj, W. Urbanczyk, T. Nasilowski, F. Berghmans, and H. Thienpont, "Highly birefringent microstructured fibers with enhanced sensitivity to hydrostatic pressure," Optics Express, vol. 18, no. 14, pp. 15113-15121, 2010.

[30] M. C. P. Huy, G. Laffont, V. Dewynter, P. Ferdinand, P. Roy, J. L. Auguste, D. Pagnoux, W. Blanc, and B. Dussardierg, "Three-hole microstructured optical fiber for efficient fiber Bragg grating refractometer," Optics Letters, vol. 32, no. 16, pp. 2390-2392, 2007.
[31] M. C. P. Huy, G. Laffont, V. Dewynter, P. Ferdinand, P. Roy, D. Pagnoux, W. Blanc, and B. Dussardierg, "Tited fiber Bragg grating photowritten in microstructured optical fiber for improved refractive index measurement," Optics Express, vol. 14, no. 22, pp. 10359-10370, 2006.

[32] A. Cusano, D. Paladino, and A. Iadicicco, "Microstructured fiber Bragg gratings," Journal of Lightwave Technology, vol. 27, no. 11, pp. 1663-1697, 2009.

[33] C. Kerbage, , R. S. Windeler, B. J. Eggleton, P. Mach, M. Dolinski, and J. A. Rogers, "Tunable devices based on dynamic positioning of micro-fluids in micro-structured optical fiber," Optics Communications, vol. 204, no. 1-6, pp. 179-184, 2002.

[34] C. Kerbage and B. J. Eggleton, "Manipulating light by microfluidic motion in microstructured optical fibers," Optical Fiber Technology, vol. 10, no. 2, pp. 133-149, 2004.

[35] L. Rindorf, J. B. Jensen, M. Dufva, L. H. Pedersen, P. E. Hoiby, and O. Bang, "Photonic crystal fiber long-period gratings for biochemical sensing," Optics Express, vol. 14, no. 18, pp. 8224-8231, 2006.

[36] Z. H. He, Y. N. Zhu, and H. Du, "Long-period gratings inscribed in air- and water-filled photonic crystal fiber for refractometric sensing of aqueous solution," Applied Physics Letters, vol. 92, no. 4, pp. 044105-1-044105-3, 2008.

[37] J. Canning, "Properties of specialist fibers and Bragg gratings for optical fiber sensors," Journal of Sensors, vol. 2009, no. 2009, pp. 1-17, 2009.

[38] M. C. P. Huy, G. Laffont, Y. Frignac, V. Dewynter-Marty, P. Ferdinand, P. Roy, J. M. Blondy, D. Pagnoux, W. Blanc, and B. Dussardier, "Fiber Bragg grating photowriting in microstructured optical fibers for refractive index measurement," Measurement Science and Technology, vol. 17, no.5, pp. 992-997, 2006.

[39] D. R. Lide, Handbook of Chemistry and Physics, 70th ed. Boca Raton: CRC Press, 1989.

[40] Y. L. Hoo, W. Jin, H. L. Ho, J. Ju, and D. N. Wang, "Gas diffusion measurement using hollow-core photonic bandgap fiber," Sensors and Actuators B: Chemical, vol. 105, no. 2, pp. 183-186, 2005.

[41] C. M. B. Cordeiro, M. A. R. Franco, G. Chesini, E. C. S. Barretto, R. Lwin, C. H. Brito Cruz, and M. C. J. Large, "Microstructured-core optical fiber for evanescent sensing applications," Optics Express, vol. 14, no. 26, pp. 13056-13066, 2006.

[42] G. Pickrell, W. Peng, and A. Wang, "Random-hole optical fiber evanescent-wave gas sensing," Optics Letters, vol. 29, no. 13, pp. 1476-1478, 2004.

[43] T. G. Euser, J. S. Y. Chen, N. J. Farrer, M. Scharrer, P. J. Sadler, and P. St. J. Russell, "Quantitative broadband chemical sensing in air-suspended 
solid-core fibers," Journal of Applied Physics, vol. 103, no. 10, pp. 103108-1-103108-7, 2008.

[44] D. S. Baer, J. B. Paul, M. Gupta, and A. O'Keefe, "Sensitive absorption measurements in the near infrared region using off-axis integrated-cavity output spectroscopy," Appliel Physics B: Chemical, vol. 75, no. 2-3, pp. 261-265, 2002.

[45] Y. L. Hoo, W. Jin, C. Z. Shi, H. L. Ho, D. N. Wang, and S. C. Ruan, "Design and modeling of a photonic crystal fiber gas sensor," Applied Optics, vol . 42, no. 18, pp. 3509-3515, 2003.

[46] Y. L. Hoo, S. J. Liu, H. L. Ho, and W. Jin, "Fast response microstructured optical fiber methane sensor with multiple side-openings," Photonics Technology Letters, vol. 22, no. 5, 296-298, 2010.

[47] L. M. Tong, R. R. Gattass, J. B. Ashcom, S. He, J. Y. Lou, M. Y. Shen, I. Maxwell, and E. Mazur, "Subwavelength-diameter silica wires for low-loss optical wave guiding," Nature, vol. 426, no. 6968, pp. 816-819, 2003.

[48] L. Zhang, J. Y. Lou, and L. M. Tong, "Micro/Nanofiber optical sensors," Photonic Sensors, vol. 1, no. 1, pp. 31-42, 2011.

[49] T. Wang, X. H. Li, F. F. Liu, W. H. Long, Z. Y. Zhang, L. M. Tong, and Y. K. Su, "Enhanced fast light in microfiber ring resonator with a Sagnac loop reflector," Optics Express, vol. 18, no. 15, pp. 16156-16161, 2010.

[50] M. Sumetsky, Y. Dulashko, J. M. Fini, and A. Hale, "Optical microfiber loop resonator," Applied Physics Letters, vol. 86, no. 16, pp. 161108-161110, 2005.
[51] X. S. Jiang, L. M. Tong, G. Vienne, X. Guo, A. Tsao, Q. Yang, and D. R. Yang, "Demonstration of optical microfiber knot resonators," Applied Physics Letters, vol. 88, no. 22, pp. 223501-223503, 2006.

[52] Y. H. Li and L. M. Tong, "Mach-Zehnder interferometers assembled with optical microfibers or nanofibers," Optics Letters, vol. 33, no. 4, pp. 303-305, 2008

[53] P. F. Wang, G. Brambilla, M. Ding, Y. Semenova, Q. $\mathrm{Wu}$, and G. Farrell, "High-sensitivity, evanescent field refractometric sensor based on a tapered, multimode fiber interference," Optics Letters, vol. 36 , no. 12, pp. 2233-2235, 2011.

[54] H. Xuan, W. Jin, and M. Zhang, " $\mathrm{CO}_{2}$ laser induced long period ratings in optical microfibers," Optics Express, vol. 17, no. 24, pp. 21882-21890, 2009.

[55] W. Liang, Y. Y. Huang, Y. Xu, R. K. Lee, and A. Yariv, "Highly sensitive fiber Bragg grating refractive index sensors," Applied Physics Letters, vol. 86, no. 15, pp. 151122-151124, 2005.

[56] A. N. Chryssis, S. M. Lee, S. B. Lee, S. S. Saini, and M. Dagenais, "High sensitivity evanescent field fiber bragg grating sensor," Photonics Technology Letters, vol. 17, no. 6, pp. 1253-1255, 2005.

[57] X. Fang, C. R. Liao, and D. N. Wang, "Femtosecond laser fabricated fiber Bragg grating in microfiber for refractive index sensing," Optics Letters, vol. 35, no. 7, pp. 1007-1009, 2010.

[58] Y. X. Liu, C. Meng, A. P. Zhang, Y. Xiao, H. K. Yu, and L. M. Tong, "Compact microfiber Bragg gratings with high-index contrast," Optics Letters, vol. 36, no. 16, pp. 3115-3117, 2011. 